\title{
NREL Highlights \\ NREL Sheds Light on Integration Costs of Variable Generation and Cost-Causation
}

RESEARCH \& DEVELOPMENT

\author{
Integration costs are generally manageable, but calculating \\ costs is challenging.
}

Renewable energy generation sources, such as wind and solar energy, provide benefits such as reduced environmental impact, lack of fuel consumption, and low and stable costs. However, their variability and uncertainty-which can change with weather conditions, time of day, and season-cause an increase in power system operating costs.

The primary costs come from additional operating reserves that are needed to ensure system reliability and the impacts on the operations of other (non-renewable) generation plants. Generally, integration costs have been found to be manageable and modest compared with electricity prices, but there is little agreement on methodologies used to determine these costs or whether these costs are measurable.

Researchers at the National Renewable Energy Laboratory (NREL) recently examined how integration studies have evolved, the analysis techniques used, common analysis mistakes, and why calculating integration costs is so challenging. Although a number of studies have assessed integration costs, calculating them correctly is challenging because it is difficult to accurately develop a baseline scenario without variable generation that properly accounts for the energy value. It is also difficult to appropriately allocate costs given the complex, nonlinear interactions between resources and loads.

Complicating matters, integration costs are not unique to variable generation. Other types of generation impose integration costs, too. For example, nuclear power plants or new, extremely inexpensive baseload units may force other baseload units to run very differently and incur integration costs, and plants that can't follow automatic generation control also incur integration costs. Integration costs could either be broadly shared, or could instead be assessed based on performance.

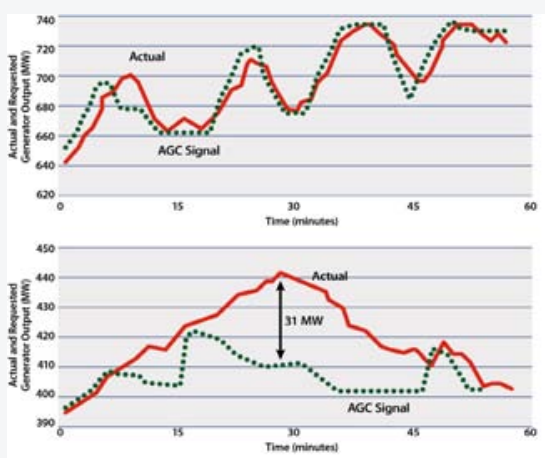

Two coal-fired generators in the Midwest illustrate a difference in ability to follow an automatic generation control signal. The upper generator is providing regulation, while the lower generator is imposing a regulation burden on the power system.

\section{Key Research Results}

\section{Achievement}

NREL analysis shows that integration costs are small and not unique to wind and solar.

\section{Key Result}

Operational changes — such as wind and solar forecasting, larger balancing areas, and fast dispatch — can reduce the costs of integrating renewable generators.

\section{Potential Impact}

Correctly assessing integration costs will inform better decision-making and aid utilities in the procurement of wind and solar energy. 